\title{
Prognostic value of lower bone mineral density in predicting adverse cardiovascular disease in Asian women
}

\author{
Jiesuck Park, ${ }^{1,2}$ Yeonyee Elizabeth Yoon (D) , ${ }^{1,2}$ Kyoung Min Kim, ${ }^{2,3}$ In-Chang Hwang, ${ }^{1,2}$ \\ Wonjae Lee, ${ }^{1,2}$ Goo-Yeong Cho (D) ${ }^{1,2}$
}

- Additional material is published online only. To view please visit the journal online (http://dx.doi.org/10.1136/ heartjnl-2020-318764).

For numbered affiliations see end of article.

\section{Correspondence to} Yeonyee Elizabeth Yoon, Department of Cardiology, Cardiovascular Center, Seoul National University Bundang Hospital, Seongnam, Republic of Korea;

yeonyeeyoon@snubh.org and Kyoung Min Kim, Division of Endocrinology and Metabolism, Department of Internal Medicine, Yongin Severance Hospital, Yonsei University College of Medicine, Yongin, Republic of Korea; kyoungmin.kim@yonsei.ac.kr

Received 2 December 2020 Revised 5 March 2021 Accepted 13 March 2021 Published Online First 7 May 2021

\section{Linked}

- http://dx.doi.org/10.1136/ heartjnl-2021-319265

Check for updates

(C) Author(s) (or their employer(s)) 2021. No commercial re-use. See rights and permissions. Published by BMJ.

To cite: Park J, Yoon YE, Kim KM, et al. Heart 2021:107:1040-1046.

\section{ABSTRACT}

Objective We investigated whether the evaluation of bone mineral density (BMD) provides independent and incremental prognostic value for predicting atherosclerotic cardiovascular disease (ASCVD) in women.

Methods A total of 12681 women aged $50-80$ years (mean, 63.0 \pm 7.8 years) who underwent dual-energy $\mathrm{X}$-ray absorptiometry were retrospectively analysed. We assessed the hazard ratio (HR) for ASCVD events (ASCVD death, non-fatal myocardial infarction and ischaemic stroke) according to the BMD or a clinical diagnosis of osteopenia or osteoporosis, with adjustment for clinical risk factors, including age, body mass index, hypertension, type 2 diabetes, hyperlipidaemia, current smoking and previous fracture. We also evaluated whether the addition of BMD or a clinical diagnosis of osteopenia or osteoporosis to clinical risk factors improved the prediction for ASCVD events.

Results In total, 468 women (3.7\%) experienced ASCVD events during follow-up (median, 9.2 years). Lower BMD at the lumbar spine, femur neck and total hip was independently associated with higher risk for ASCVD events (adjusted HR per 1-standard deviation decrease in BMD: 1.16, $p<0.001 ; 1.29, p<0.001$; $1.38, p<0.001$; respectively). A clinical diagnosis of osteoporosis was also independently associated with higher risk for ASCVD events (adjusted HR: $1.79, p<0.001)$. The addition of BMD or a clinical diagnosis of osteopenia or osteoporosis to clinical risk factors demonstrated significant incremental value in discriminating ASCVD events (addition of total hip BMD, $p$ for difference $<0.001$ ).

Conclusion The evaluation of BMD provides independent and incremental prognostic value for ASCVD in women and thus may improve risk stratification in women.

\section{INTRODUCTION}

Atherosclerosis and osteoporosis are major agerelated diseases, predominantly contributing to significant morbidity and mortality in women. ${ }^{1}$ Atherosclerosis comprises a multifactorial process resulting in atherosclerotic cardiovascular disease (ASCVD), which is the leading cause of death globally. ${ }^{2}$ Osteoporosis, characterised by low bone mineral density (BMD) and bone structure deterioration, reflects a condition of impaired bone strength and, thus, higher risk of fracture, leading to a loss of independence and higher financial burden. ${ }^{3}$ Previous epidemiologic studies indicate the coincidental occurrence of atherosclerosis and osteoporosis, ${ }^{4}$ suggesting a potential association between these diseases. ${ }^{14}$ However, most of the available data were derived from cross-sectional studies, and the prognostic value of BMD in predicting ASCVD has not been well evaluated. Furthermore, there is conflicting evidence regarding an independent association between osteoporosis and atherosclerosis, which both occur during the ageing process and share common risk factors. ${ }^{14-7}$

As the population-adjusted risk of ASCVD mortality is higher in women $(21 \%)$ than in men $(15 \%)$, traditional risk factors may differentially affect men and women. ${ }^{8}$ Additionally, as the performance of the conventional prediction algorithm is limited in women, ${ }^{910}$ new strategies that go beyond the measurement of conventional risk factors are needed to better identify women at higher risk of ASCVD. Given that millions of women are screened for osteoporosis using dual-energy X-ray absorptiometry (DXA), potential associations between low bone mass and ASCVD in women would provide an opportunity to improve the risk stratification of women without any additional costs. Therefore, we investigated whether the evaluation of BMD provides independent and incremental prognostic value for ASCVD events in a large cohort of women who underwent DXA.

\section{METHODS}

\section{Study population}

The medical records of consecutive women aged 50-80 years who underwent DXA as a screening for osteoporosis at Seoul National University Bundang Hospital between 2005 and 2014 were retrospectively reviewed. For women who underwent repeated DXAs during the study period, only the first DXA was included. Among this initial cohort $(n=14333)$, we excluded patients with a history of myocardial infarction (MI) or coronary revascularisation $(n=285)$, malignant tumour $(n=870)$, chronic kidney disease $(n=89)$ and previous osteoporosis treatment $(n=11)$. Additionally, patients without complete BMD measurements at the lumbar spine, femur neck and total hip $(n=121)$ and those without follow-up data $(n=276)$ were excluded. Finally, a total of 12681 women were included for analysis (online supplemental figure 1). 


\section{Patient and public involvement}

Patients or the public were not involved in the design, or conduct, or reporting, or dissemination plans of the study.

\section{Ascertainment of risk factors}

Baseline characteristics were acquired by a dedicated review of the electronic health records of our institution, which was fully digitalised from its foundation. ${ }^{11}$ The diagnostic definitions for the clinical risk factors were as previously validated: hypertension (HTN) was defined as the presence of a diagnosis of HTN, blood pressure higher than $140 / 90 \mathrm{~mm} \mathrm{Hg}$, or anti-hypertensive medication use within 6 months of the index DXA study date; type 2 diabetes mellitus (T2DM) was defined as the presence of a diagnosis of T2DM, fasting plasma glucose level of $126 \mathrm{mg} /$ $\mathrm{dL}$ or higher, haemoglobin A1c level of $6.5 \%$ or higher, or antidiabetic medication use within 6 months of the index date; and hyperlipidaemia (HL) was defined as the presence of a diagnosis of HL, total cholesterol level of $240 \mathrm{mg} / \mathrm{dL}$ or higher, low-density lipoprotein cholesterol level of $160 \mathrm{mg} / \mathrm{dL}$ or higher, or treatment with statins within 6 months of the index date. ${ }^{12-16}$ Body mass index (BMI) was calculated by the weight $(\mathrm{kg})$ divided by the square of the height $\left(\mathrm{m}^{2}\right)$, required during the DXA scan. The predicted 10-year ASCVD risk was estimated using the Korean Risk Prediction Model, which is a recalibration of the Pooled Cohort Equation specifically for the Korean population. ${ }^{17}$

\section{Measurement of BMD}

BMD was measured at the lumbar spine, femoral neck and total hip using a single DXA scanner (Lunar Prodigy; GE, Madison, Wisconsin, USA), following the manufacturer's protocol. ${ }^{18}$ For the measurement of BMD at the lumbar spine, vertebrae with a BMD higher or lower than 1 standard deviation (SD) of that in adjacent vertebrae were excluded from analyses, in accordance with the International Society for Clinical Densitometry rules for excluding individual vertebrae. ${ }^{19} \mathrm{~T}$-scores were calculated for each measurement site, using the standard reference for Asian populations provided by the manufacturer. Osteopenia was defined as a BMD T-score between -1.0 and -2.5 , and osteoporosis was defined as a T-score below -2.5 , in accordance with World Health Organization guidelines. ${ }^{20}$ The precision error (per cent coefficient of variation) was less than $2 \%$ for the lumbar spine, femoral neck, and total hip BMD.

\section{Study outcomes}

The primary outcome was the ASCVD events of ASCVD death, non-fatal MI and non-fatal ischaemic stroke. We also assessed ASCVD death as a secondary outcome. Data regarding the date and cause of death were acquired from the Cause of Death Statistics from Statistics Korea, which collected data from death notifications filed at local administration offices and medical certificates of death mandatorily issued by physicians. Follow-up data for non-fatal MI and ischaemic stroke were obtained via a dedicated review of electronic health records by an independent investigator who was blinded to the baseline BMDs. The date of the DXA scan was defined as the index date of follow-up. Subjects were censored at the occurrence of outcome events or at the last date of the follow-up period for those who were lost to follow-up or had been transferred-out.

\section{Statistical analysis}

Cox proportional hazard regression modelling was used to estimate hazard ratios (HRs) for ASCVD events according to the per 1-SD decrease in BMD for each measurement site (lumbar spine, femur neck and total hip), or a clinical diagnosis of osteopenia or osteoporosis based on the BMD T-score for each measurement site, using women with normal T-scores as the reference group. We also evaluated the HRs for ASCVD according to a clinical diagnosis of osteopenia or osteoporosis based on the combined T-score of the three measurement sites (referred to as the 'combined T-score'). HRs were estimated with adjustment for age only, and for age and BMI, HTN, T2DM, HL, current smoking and previous fracture (referred to as 'other clinical factors'). We additionally adjusted for competing mortality risks.

Subgroup analyses were conducted to evaluate whether the predictive value of BMD differed according to the presence or absence of each clinical risk factor (age $\geq 70$ years, BMI $\geq 25$ $\mathrm{kg} / \mathrm{m}^{2}$, current smoking, HTN, T2DM, HL, previous fracture). Additionally, we evaluated whether a clinical diagnosis of osteopenia or osteoporosis was associated with an increased risk of ASCVD even in a low-risk subgroup of women aged $<70$ years with no documented clinical risk factors (BMI $\geq 25 \mathrm{~kg} /$ $\mathrm{m}^{2}$, current smoking, HTN, T2DM, HL, previous fracture). Furthermore, considering the varied follow-up duration due to the retrospective nature of the study, we performed a sensitivity analysis, excluding women who were censored without outcome events before the median follow-up.

The incremental prognostic value of BMD was calculated by defining prediction models with the sequential addition of age (Model 1), other clinical risk factors (Model 2), and BMD (Model 3) or a clinical diagnosis of osteopenia or osteoporosis (Model 4). Model calibration was evaluated by calibration plot and Brier score comparing the predicted probabilities of the primary outcome against observed probabilities (online supplemental figure 2). Model discriminatory performance was assessed by the Harrell's concordance statistic (c-statistic), and the integrated discrimination improvement (IDI) and categorical net reclassification index (NRI) were used to compare the reclassification performance among models. We applied random permutation methods, selecting random subsamples from the whole population 1000 times, for a robust calculation of CIs. We additionally compared the performance of the best model in predicting ASCVD events to that of the 10-year ASCVD risk.

All statistical analyses were performed using $\mathrm{R}$ software, V.3.6.1 (R Development Core Team, Vienna, Austria). Two-sided $\mathrm{p}$ values $<0.05$ were considered statistically significant.

\section{RESULTS}

\section{Clinical characteristics of the study population}

Detailed clinical characteristics of the 12681 women (mean age, $63.0 \pm 7.8$ years; mean BMI, $24.1 \pm 3.2 \mathrm{~kg} / \mathrm{m}^{2}$ ) are summarised in table 1. Baseline characteristics stratified by osteopenia and osteoporosis are shown in online supplemental table 1 . None of the study participants had been prescribed hormone replacement therapy for more than 180 days within 6 months before and after the index DXA date.

\section{Prediction of ASCVD events}

During a median follow-up of 9.2 years (IQR, 3.5-11.5 years), $468(3.7 \%)$ women experienced the primary outcome (ASCVD events). Age, current smoking and T2DM were independently associated with higher risk of ASCVD in a model with age, other clinical risk factors and BMD (each site) (online supplemental table 2). BMDs of the lumbar spine, femur neck and total hip were significant independent risk predictors of ASCVD when adjusted for age and other clinical risk factors (table 2). The diagnoses of osteopenia and osteoporosis, based on the T-scores 


\section{Table 1 Baseline characteristics}

\begin{tabular}{|c|c|}
\hline & Total study population ( $n=12681)$ \\
\hline Age, mean $(S D)$, years & $63.0(7.8)$ \\
\hline $\mathrm{BMI}$, mean (SD), kg/m² & $24.1(3.2)$ \\
\hline Current smoking, N (\%) & $208(1.6)$ \\
\hline Hypertension, N (\%) & $3811(30.1)$ \\
\hline Type 2 diabetes mellitus, N (\%) & $1336(10.5)$ \\
\hline Hyperlipidaemia, N (\%) & $4370(34.5)$ \\
\hline Previous fracture, N (\%) & $481(3.8)$ \\
\hline 10-year ASCVD risk score, mean (SD) & $9.6(6.7)$ \\
\hline$<7.5 \%, \mathrm{~N}(\%)$ & $6174(48.7)$ \\
\hline$\geq 7.5 \%, \mathrm{~N}(\%)$ & $6507(51.3)$ \\
\hline Lumber BMD, mean (SD), g/cm² & $0.985(0.173)$ \\
\hline Lumber T-score, mean (SD) & $-1.0(1.4)$ \\
\hline \multicolumn{2}{|l|}{ Diagnosis based on the lumbar T-score } \\
\hline Normal, N (\%) & $5660(44.6)$ \\
\hline Osteopenia, N (\%) & $5014(39.5)$ \\
\hline Osteoporosis, N (\%) & $2007(15.8)$ \\
\hline Femur neck BMD, $\mathrm{g} / \mathrm{cm}^{2}$ & $0.779(0.128)$ \\
\hline Femur neck T-score, mean (SD) & $-1.0(1.1)$ \\
\hline \multicolumn{2}{|l|}{ Diagnosis based on the femur neck T-score } \\
\hline Normal, N (\%) & $5837(46.0)$ \\
\hline Osteopenia, N (\%) & $5845(46.1)$ \\
\hline Osteoporosis, N (\%) & $999(7.9)$ \\
\hline Total hip BMD, $\mathrm{g} / \mathrm{cm}^{2}$ & $0.845(0.139)$ \\
\hline Total hip T-score, mean (SD) & $-0.7(1.2)$ \\
\hline \multicolumn{2}{|l|}{ Diagnosis based on the total hip T-score } \\
\hline Normal, N (\%) & $7229(57.0)$ \\
\hline Osteopenia, N (\%) & $4621(36.4)$ \\
\hline Osteoporosis, N (\%) & $831(6.6)$ \\
\hline \multicolumn{2}{|c|}{ Diagnosis based on the combined T-score of the three measurement sites } \\
\hline Normal, N (\%) & $3985(31.4)$ \\
\hline Osteopenia, N (\%) & $6214(49.0)$ \\
\hline Osteoporosis, N (\%) & $2482(19.6)$ \\
\hline
\end{tabular}

at each measurement site, also demonstrated increasing associations with ASCVD events (table 2). In particular, the clinical diagnosis of osteoporosis by the T-score at any of the three measurement sites was independently associated with ASCVD events, even after adjustment for age and other clinical risk factors (table 2). When the presence of osteopenia and osteoporosis was defined by the combined T-score, the clinical diagnosis of osteoporosis based on the combined T-score was also independently associated with ASCVD events after covariate adjustment (table 2). Regarding the secondary outcome, 237 (1.9\%) women experienced ASCVD death during follow-up. Lower $\mathrm{BMDs}$ or the diagnosis of osteoporosis were associated with significantly higher risk of ASCVD death (table 3).

Lower BMDs or a clinical diagnosis of osteoporosis maintained an independent association with higher risk of ASCVD events and ASCVD death after adjustment for competing mortality risks (online supplemental table 3). Additionally, in women with follow-up longer than the median follow-up period, consistent results were obtained, with higher risk for ASCVD events linked to lower BMD or a clinical diagnosis of osteoporosis (online supplemental table 4).

The subgroup analyses according to the presence or absence of each clinical risk factor revealed that 1-SD decreases in femur neck and total hip BMDs were significantly associated with higher risk for ASCVD events for almost all risk factors (figure 1). Furthermore, in a low-risk subgroup ( $n=3$ 878), a diagnosis of osteoporosis based on the femur neck or total hip T-score was associated with higher risk for ASCVD events (online supplemental table 5). Additionally, a diagnosis of osteoporosis from any measurement site was associated with higher risk for ASCVD death (online supplemental table 5).

\section{Incremental prognostic value of BMD}

Model performance in the discrimination of ASCVD events is shown in table 4 . The addition of other clinical risk factors to Model 1 resulted in a significant improvement in the discrimination for ASCVD events (Model 2). The addition of the BMD of each measurement site to Model 2 resulted in a significant improvement in model performance (Model 3). The addition of a clinical diagnosis of osteopenia or osteoporosis based on the T-score (each site or combined) to Model 2 also resulted in a significant improvement in model performance (Model 4). Furthermore, the incremental prognostic value of BMD, or a clinical diagnosis of osteopenia or osteoporosis, was also confirmed by significant improvements in the NRI and IDI (table 4). Overall, the total hip area provided the strongest improvement in risk stratification for ASCVD events. When we additionally compared the discrimination ability of the highest performing model (Model 4 with a clinical diagnosis based on the total hip BMD) with that of the 10-year ASCVD risk, Model 4 showed significantly better discrimination (figure 2).

\section{DISCUSSION}

Although a potential association between osteoporosis and ASCVD has been proposed, the prognostic value of BMD in predicting future ASCVD has not been well evaluated. In the present study, we evaluated the value of BMD in predicting future ASCVD, using a large cohort of consecutive women who underwent DXA. We found that low BMD has independent and incremental value in predicting future ASCVD in women.

To date, mounting epidemiological evidence suggests an association between decreased BMD and ASCVD. Previous studies have reported a higher prevalence of atherosclerotic coronary artery disease in patients with lower BMD and vice versa. ${ }^{21} 22 \mathrm{~A}$ higher incidence of fracture events in patients with atherosclerosis has also been reported. ${ }^{23}$ Furthermore, the beneficial effect of anti-osteoporotic drugs on atherogenesis and lipid profiles ${ }^{24}$ is consistent with a potential link between bone and vasculature biologies. However, most previous studies were limited by a cross-sectional observational design and small number of study participants. Additionally, conflicting data demonstrating an age-dependent association, or even no association, between low BMD and atherosclerosis have been reported. ${ }^{25}$ Although a recent meta-analysis reported a strong association between decreased BMD and risk for atherosclerotic vascular abnormalities, independent of age, sex and other cardiovascular risk factors, the meta-analysis comprised mostly case-control and cross-sectional cohort studies. ${ }^{4}$ To our knowledge, the present study is the largest longitudinal study evaluating the association between BMD and risk for ASCVD. Lower BMD was significantly associated with higher risk for ASCVD events, independent of age and other clinical risk factors. Additionally, a clinical diagnosis of osteoporosis consistently provided independent prognostic value for ASCVD events. These results suggest an independent association between decreased BMD and ASCVD, supporting the potential role of BMD as a novel risk marker for future ASCVD in women.

Several potential mechanisms have been proposed to explain a link between atherosclerosis and osteoporosis. Chronic 
Table 2 Adjusted HRs of BMD values and a clinical diagnosis of osteopenia or osteoporosis for atherosclerotic cardiovascular disease events

\begin{tabular}{|c|c|c|c|c|c|c|}
\hline \multicolumn{3}{|l|}{ Risk factors } & $\begin{array}{l}\text { Age-adjusted } \mathrm{HR}^{*} \\
(95 \% \mathrm{Cl})\end{array}$ & $P$ value & $\begin{array}{l}\text { Fully adjusted HRt } \\
(95 \% \mathrm{Cl})\end{array}$ & $P$ value \\
\hline \multicolumn{7}{|c|}{$B M D$ as a continuous variable } \\
\hline \multicolumn{3}{|c|}{ Lumbar BMD (per 1-SD decrease) } & 1.14 (1.07 to 1.22$)$ & $<0.001$ & 1.16 (1.07 to 1.24$)$ & $<0.001$ \\
\hline \multicolumn{3}{|c|}{ Femur neck BMD (per 1-SD decrease) } & 1.30 (1.16 to 1.45$)$ & $<0.001$ & $1.29(1.15$ to 1.45$)$ & $<0.001$ \\
\hline \multicolumn{3}{|c|}{ Total hip BMD (per 1-SD decrease) } & 1.35 (1.23 to 1.48$)$ & $<0.001$ & $1.38(1.25$ to 1.53$)$ & $<0.001$ \\
\hline \multicolumn{7}{|c|}{ Diagnoses of osteopenia and osteoporosis based on the T-score } \\
\hline Lumbar T-score & Number of women & Event, $\mathbf{n}(\%)$ & & & & \\
\hline Normal & 5660 & $144(2.5)$ & 1 (reference) & & 1 (reference) & \\
\hline Osteopenia & 5014 & $171(3.4)$ & 0.98 (0.78 to 1.22$)$ & 0.828 & 0.99 (0.79 to 1.24$)$ & 0.906 \\
\hline \multirow{2}{*}{ Osteoporosis } & 2007 & $153(7.6)$ & 1.62 (1.27 to 2.06$)$ & $<0.001$ & 1.67 (1.30 to 2.14$)$ & $<0.001$ \\
\hline & \multicolumn{6}{|c|}{ Femur neck T-score } \\
\hline Normal & 5837 & $109(1.9)$ & 1 (reference) & & 1 (reference) & \\
\hline Osteopenia & 5845 & $245(4.2)$ & 1.24 (0.97 to 1.58$)$ & 0.084 & 1.27 (0.99 to 1.62$)$ & 0.058 \\
\hline \multirow{2}{*}{ Osteoporosis } & 999 & $114(11.4)$ & 2.25 (1.66 to 3.05$)$ & $<0.001$ & 2.27 (1.66 to 3.11$)$ & $<0.001$ \\
\hline & \multicolumn{6}{|c|}{ Total hip T-score } \\
\hline Normal & 7229 & $150(2.1)$ & 1 (reference) & & 1 (reference) & \\
\hline Osteopenia & 4621 & $210(4.5)$ & 1.30 (1.04 to 1.63$)$ & 0.022 & 1.37 (1.09 to 1.72$)$ & 0.006 \\
\hline Osteoporosis & 831 & $108(13.0)$ & 2.70 (2.03 to 3.58$)$ & $<0.001$ & 2.95 (2.18 to 3.98 ) & $<0.001$ \\
\hline \multicolumn{7}{|l|}{ Combined T-score } \\
\hline Normal & 3985 & $73(1.8)$ & 1 (reference) & & 1 (reference) & \\
\hline Osteopenia & 6214 & $199(3.2)$ & 0.98 (0.74 to 1.30$)$ & 0.903 & 0.99 (0.75 to 1.31$)$ & 0.947 \\
\hline Osteoporosis & 2482 & $196(7.9)$ & 1.73 (1.28 to 2.34$)$ & $<0.001$ & 1.79 (1.32 to 2.44$)$ & $<0.001$ \\
\hline
\end{tabular}

inflammation and accumulative oxidative stress play key roles in both aging-related bone loss and atherogenesis. ${ }^{1}$ Therefore, these shared common pathogeneses might contribute to the coincidental occurrence of osteoporosis and atherosclerosis. Parathyroid hormone, a principal regulator of calcium and phosphorus balance, plays a major physiologic role in bone homeostasis. Disturbed calcium and phosphorus metabolism are also known to accelerate both bone loss and atherogenesis. ${ }^{1415}$ Furthermore, osteogenic mediators released during bone loss, including osteoprotegerin, osteocalcin and sclerostin, are known to have direct effects on vasculature biology and promote atherosclerosis. ${ }^{1}$ Additionally, sex hormones, particularly oestrogen, play critical roles in regulating bone biology and the vasculature and might contribute to the link between these two conditions. ${ }^{26}$ However,

Table 3 ASCVD death according to BMD values and a clinical diagnosis of osteopenia or osteoporosis

\begin{tabular}{|c|c|c|c|c|c|}
\hline \multicolumn{2}{|l|}{ Risk factors } & $\begin{array}{l}\text { Age-adjusted HR* } \\
(95 \% \mathrm{Cl})\end{array}$ & $P$ value & $\begin{array}{l}\text { Fully adjusted HRT } \\
(95 \% \mathrm{Cl})\end{array}$ & $P$ value \\
\hline \multicolumn{6}{|c|}{$B M D$ as a continuous variable } \\
\hline \multicolumn{2}{|c|}{ Lumbar BMD (per 1-SD decrease) } & 1.28 (1.16 to 1.41$)$ & $<0.001$ & 1.24 (1.12 to 1.38$)$ & $<0.001$ \\
\hline \multicolumn{2}{|c|}{ Femur neck BMD (per 1-SD decrease) } & $1.67(1.43$ to 1.96$)$ & $<0.001$ & $1.57(1.33$ to 1.85$)$ & $<0.001$ \\
\hline \multicolumn{2}{|c|}{ Total hip BMD (per 1-SD decrease) } & 1.76 (1.55 to 2.01$)$ & $<0.001$ & 1.73 (1.50 to 1.99$)$ & $<0.001$ \\
\hline \multicolumn{6}{|c|}{ Diagnoses of osteopenia and osteoporosis based on the T-score } \\
\hline Lumbar T-score & Event, $\mathbf{n}(\%)$ & & & & \\
\hline Normal & $58(1.0)$ & 1 (reference) & & 1 (reference) & \\
\hline Osteopenia & $77(1.5)$ & 1.00 (0.71 to 1.41$)$ & 0.991 & $0.99(0.70$ to 1.40$)$ & 0.975 \\
\hline \multirow{2}{*}{$\begin{array}{l}\text { Osteoporosis } \\
\text { Femur neck T-sco }\end{array}$} & $102(5.1)$ & 2.17 (1.55 to 3.03$)$ & $<0.001$ & 1.99 (1.40 to 2.83$)$ & $<0.001$ \\
\hline & \multicolumn{5}{|c|}{ Femur neck T-score } \\
\hline Normal & $35(0.6)$ & 1 (reference) & & 1 (reference) & \\
\hline Osteopenia & $123(2.1)$ & 1.54 (1.04 to 2.30$)$ & 0.032 & 1.52 (1.02 to 2.26$)$ & 0.039 \\
\hline \multirow[t]{2}{*}{ Osteoporosis } & $79(7.9)$ & 3.32 (2.12 to 5.21$)$ & $<0.001$ & 2.93 (1.85 to 4.65$)$ & $<0.001$ \\
\hline & \multicolumn{5}{|c|}{ Total hip T-score } \\
\hline Normal & $45(0.6)$ & 1 (reference) & & 1 (reference) & \\
\hline Osteopenia & $112(2.4)$ & 1.96 (1.36 to 2.82 ) & $<0.001$ & 1.98 (1.38 to 2.86$)$ & $<0.001$ \\
\hline Osteoporosis & $80(9.6)$ & 5.09 (3.37 to 7.68 ) & $<0.001$ & 4.90 (3.17 to 7.56$)$ & $<0.001$ \\
\hline \multicolumn{6}{|l|}{ Combined T-score } \\
\hline Normal & $24(0.6)$ & 1 (reference) & & 1 (reference) & \\
\hline Osteopenia & $87(1.4)$ & 1.04 (0.65 to 1.66$)$ & 0.861 & 1.03 (0.65 to 1.65$)$ & 0.890 \\
\hline Osteoporosis & $126(5.1)$ & 2.34 (1.46 to 3.77$)$ & $<0.001$ & 2.14 (1.32 to 3.48 ) & 0.002 \\
\hline
\end{tabular}




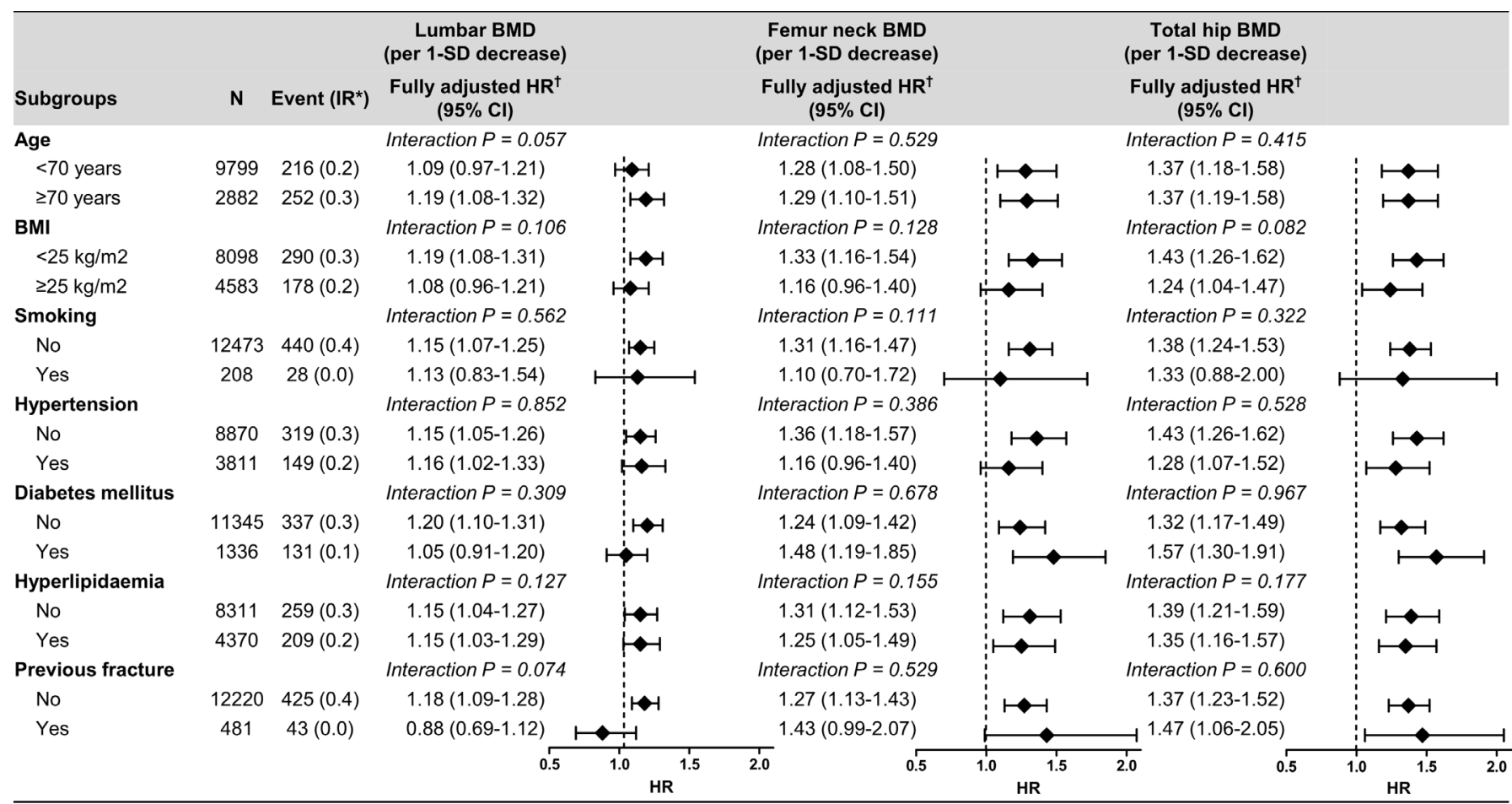

* per 100 person-year

† HRs were adjusted for age, BMI, smoking, hypertension, diabetes mellitus, hyperlipidaemia, and previous fracture

Figure 1 Subgroup analyses for the risk of atherosclerotic cardiovascular disease per 1-SD decrease in BMD

Subgroup analyses according to each clinical risk factor reveal that 1-SD decreases in the femur neck and total hip BMD are significantly associated with higher risk for atherosclerotic cardiovascular disease events after covariate adjustment. BMD, bone mineral density; BMI, body mass index; CI, confidence interval; IR, incidence rate.

the exact mechanism between lower BMD and higher risk for atherogenesis is not yet fully understood and remains to be further addressed.

The current approach based on the 10-year ASCVD risk, estimated by risk-scoring algorithms such as the Pooled Cohort Equation, has limited accuracy in identifying women at high risk of developing ASCVD. ${ }^{10}$ Although the risk prediction could be improved by additional test data, such as the coronary artery calcium score, ${ }^{27}$ widespread implementation of coronary artery calcium scanning has been unsuccessful because of the perceived risks of radiation exposure and cost. In contrast, DXA is widely used to screen for osteopenia and osteoporosis in asymptomatic women. Therefore, the present study results of an improved ASCVD risk prediction by assessing BMD suggest an opportunity to screen women for ASCVD in a manner that is effective, efficient and broad-reaching. Moreover, the risk for ASCVD could even be further stratified according to BMD in a subgroup of relatively young women with no risk factors. This result supports a potential role of BMD in identifying apparently healthy women potentially at increased ASCVD risk. However, further evaluation is required to determine the degree to which BMD refines the risk assessment compared with that with more direct imaging tests of the cardiovascular system, such as carotid ultrasonography or coronary artery calcium imaging. Additionally, comparison with the predictive value of novel risk markers, such as high-sensitivity $\mathrm{C}$ reactive protein or lipoprotein(a), would be valuable, given that these risk markers are also expected to improve the risk stratification for ASCVD. ${ }^{28} 29$

Table 4 Comparisons among prediction models for the risk of atherosclerotic cardiovascular disease events

\begin{tabular}{|c|c|c|c|c|c|c|c|}
\hline & & c-index $(95 \% \mathrm{Cl})$ & P for difference & NRI $(95 \% \mathrm{Cl})$ & $P$ value & IDI (95\% Cl) & $P$ value \\
\hline \multirow[t]{2}{*}{ Model 1} & Age & & & & & & \\
\hline & & 0.754 (0.747 to 0.761$)$ & & & & & \\
\hline \multirow{2}{*}{$\begin{array}{l}\text { Model } 2 \\
\text { (vs Model 1) }\end{array}$} & Model 1+clinical risk factors & & & & & & \\
\hline & & $0.783(0.776$ to 0.790$)$ & $<0.001$ & $0.049(0.016$ to 0.081$)$ & 0.003 & 0.008 (0.004 to 0.012$)$ & $<0.001$ \\
\hline \multirow{4}{*}{$\begin{array}{l}\text { Model } 3 \\
\text { (vs Model 2) }\end{array}$} & Model $2+B M D$ as a continuous variable & & & & & & \\
\hline & Lumbar BMD & 0.786 (0.780 to 0.793$)$ & 0.001 & 0.028 (0.011 to 0.046$)$ & 0.002 & 0.001 (0.000 to 0.003$)$ & 0.140 \\
\hline & Femur neck BMD & 0.786 (0.780 to 0.793$)$ & 0.005 & 0.036 (0.017 to 0.055$)$ & $<0.001$ & 0.002 (0.001 to 0.004$)$ & 0.018 \\
\hline & Total hip BMD & 0.790 (0.783 to 0.796$)$ & $<0.001$ & $0.036(0.013$ to 0.058$)$ & 0.002 & $0.004(0.002$ to 0.007$)$ & $<0.001$ \\
\hline \multirow{5}{*}{$\begin{array}{l}\text { Model } 4 \\
\text { (vs Model 2) }\end{array}$} & Model 2+diagnosis of osteopenia or ost & eoporosis based on the T- & & & & & \\
\hline & Lumbar T-score & 0.787 (0.781 to 0.795$)$ & 0.003 & 0.030 (0.009 to 0.052$)$ & 0.006 & 0.002 (0.001 to 0.004$)$ & 0.038 \\
\hline & Femur neck T-score & 0.790 (0.783 to 0.797$)$ & 0.004 & 0.025 (0.006 to 0.044$)$ & 0.008 & $0.003(0.001$ to 0.005$)$ & 0.006 \\
\hline & Total hip T-score & 0.793 (0.786 to 0.799$)$ & $<0.001$ & 0.039 (0.015 to 0.062$)$ & 0.001 & 0.005 (0.002 to 0.008$)$ & $<0.001$ \\
\hline & Combined T-score & 0.790 (0.784 to 0.797$)$ & $<0.001$ & $0.046(0.024$ to 0.068$)$ & $<0.001$ & 0.002 (0.001 to 0.004$)$ & 0.016 \\
\hline
\end{tabular}



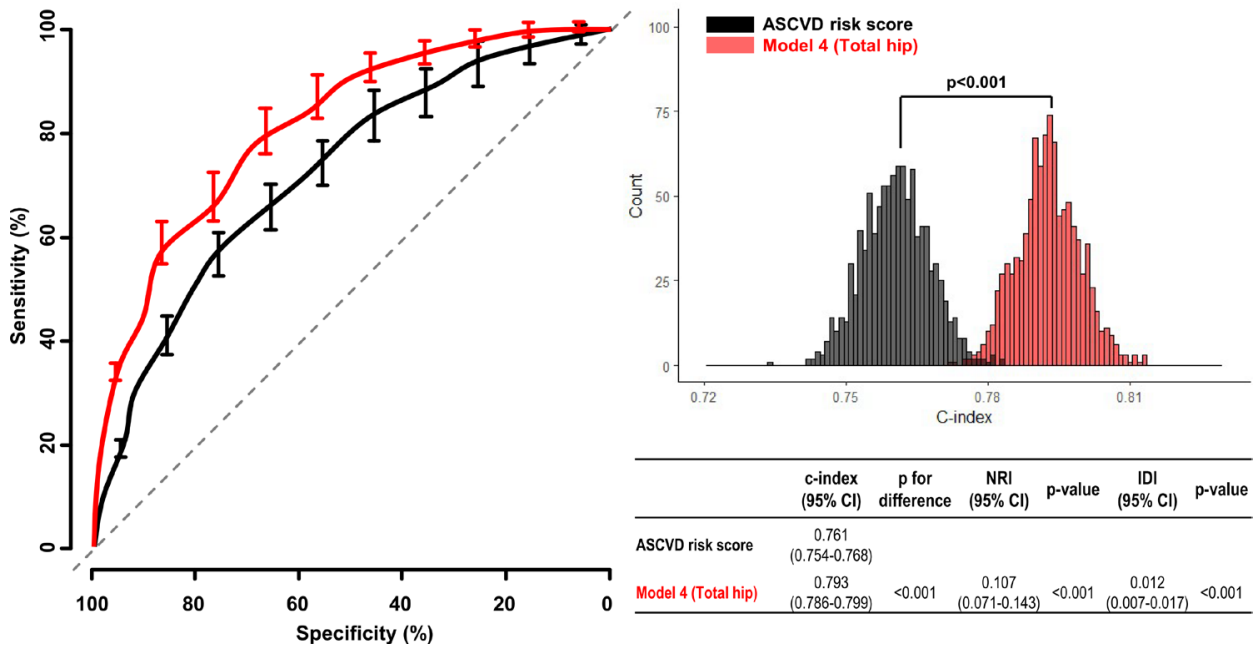

Figure 2 Comparison of prediction performance for ASCVD events between the BMD model and ASCVD risk score

The addition of a clinical diagnosis of osteopenia or osteoporosis by total hip BMD to age and other clinical risk factors demonstrates a significant improvement in model performance for predicting ASCVD events over that of the ASCVD risk score. ASCVD, atherosclerotic cardiovascular disease; $\mathrm{BMD}$, bone mineral density; Cl, confidence interval; IDI, integrated discrimination improvement; NRI, net reclassification index

Furthermore, before the widespread utilisation of BMD to facilitate personalised decision-making, randomised controlled trials of an integrated screening and targeted prevention strategy are required.

The study design introduced several limitations. First, the study results should be interpreted in the context of the limitations inherent to its retrospective medical record review study design. Since we could not obtain reliable data regarding possible important confounders, such as menopause, we only included women over the age of 50 years. Therefore, only women with postmenopausal or perimenopausal status were included in the study analysis. We also could not precisely assess physical activity levels or steroid use, which can affect both BMD and ASCVD risk. Further prospective studies that include these potential confounders are required to confirm the independent association between low BMD and risk for ASCVD. Second, we did not assess any parameters related to inflammation, oxidative stress or disturbed calcium metabolism, which are potential mediators between bone and vasculature. Whether and how these parameters affect the observed association between low BMD and ASCVD risk should be addressed in further studies. Third, as the present study comprised a cohort of women from a single tertiary medical centre in South Korea, a selection bias must be considered. Although the prevalence of osteoporosis in the current study was very similar to that from a nationwide survey of Korean residents, ${ }^{30}$ the study population may not be fully representative of the general population, and the results cannot be generalised to men or other ethnic groups. Additionally, because the study participants had a low burden of clinical risk factors, suggesting a relatively healthy population, our results may not be suitable for those with high-risk profiles. However, the strength of this study is that it is currently the largest to evaluate the association between BMD and risk for ASCVD events. Furthermore, because of its longitudinal design, we were able to evaluate whether refining the risk stratification by adding the BMD would improve the discrimination for future ASCVD. We believe that this study sets the stage for an outcome trial to evaluate whether assessing BMD in women translates into long-term clinical benefits.

\section{Key messages}

What is already known on this subject?

- Despite a potential link between osteoporosis and atherosclerotic cardiovascular disease (ASCVD), the independent and incremental prognostic value of bone mineral density (BMD) in predicting future ASCVD has not been well evaluated.

\section{What might this study add?}

- In this retrospective observational study comprising 12 681 women aged 50 years and older, lower BMD and a diagnosis of osteoporosis were independently associated with higher risk of ASCVD. The addition of BMD or a clinical diagnosis of osteoporosis or osteopenia to clinical risk factors demonstrated significant improvement in the discrimination for ASCVD events.

How might this impact on clinical practice?

- Considering that dual-energy $\mathrm{X}$-ray absorptiometry is widely used to screen for osteopenia and osteoporosis in asymptomatic women, the significant association between BMD and higher risk of ASCVD provides an opportunity for large-scale ASCVD risk assessment in women without additional cost and radiation exposure.

\section{CONCLUSIONS}

The evaluation of BMD provides predictive value for ASCVD events in women. Moreover, it provides independent and incremental prognostic value over that for age and other clinical risk factors. Further studies are warranted to determine whether the evaluation of BMD translates into long-term clinical benefits in women.

\section{Author affiliations}

${ }^{1}$ Department of Cardiology, Cardiovascular Center, Seoul National University Bundang Hospital, Seongnam, Gyeonggi-do, Republic of Korea

${ }^{2}$ Department of Internal Medicine, Seoul National University College of Medicine, Seoul, Republic of Korea 
${ }^{3}$ Division of Endocrinology and Metabolism, Department of Internal Medicine, Yongin Severance Hospital, Yonsei University College of Medicine, Yongin, Republic of Korea

Contributors JP, YEY, KMK, I-CH, WL and G-YC developed the study concept and design. JP, YEY and KMK contributed to the acquisition, analysis and interpretation of data. JP conducted the statistical analysis. JP, YEY and KMK wrote the first draft of the manuscript. All authors contributed to the critical revision and approval of the final manuscript. YEY and KMK obtained the funding for the study data and analysis. All authors had full access to the data in the study and can take responsibility for the integrity of the data and the accuracy of the data analysis.

Funding This research was supported by a research grant from Seoul National University Bundang Hospital (Grant No 13-2018-001) to YEY and KMK and a research grant from Hanmi Pharm. Co, Ltd (Grant No 06-2018-299) to YEY. The funders had no role in the design and conduct of the study, the analysis and interpretation of the data, or the review or approval of the manuscript.

Competing interests None declared.

Patient consent for publication Not required.

Ethics approval Our institutional review board approved this retrospective study and waived the requirement for informed consent (IRB No B1806-474-001).

Provenance and peer review Not commissioned; externally peer reviewed.

Data availability statement Data are available on reasonable request by contacting the corresponding authors (yeonyeeyoon@snubh.org; kyoungmin.kim@ yonsei.ac.kr).

Supplemental material This content has been supplied by the author(s). It has not been vetted by BMJ Publishing Group Limited (BMJ) and may not have been peer-reviewed. Any opinions or recommendations discussed are solely those of the author(s) and are not endorsed by BMJ. BMJ disclaims all liability and responsibility arising from any reliance placed on the content. Where the content includes any translated material, BMJ does not warrant the accuracy and reliability of the translations (including but not limited to local regulations, clinical guidelines, terminology, drug names and drug dosages), and is not responsible for any error and/or omissions arising from translation and adaptation or otherwise.

\section{ORCID iDs}

Yeonyee Elizabeth Yoon http://orcid.org/0000-0002-8479-9889

Goo-Yeong Cho http://orcid.org/0000-0002-7067-5535

\section{REFERENCES}

1 London GM. Bone-vascular cross-talk. J Nephrol 2012;25:619-25.

2 Libby P, Buring JE, Badimon L, et al. Atherosclerosis. Nat Rev Dis Primers 2019;5:56.

3 Kling JM, Clarke BL, Sandhu NP. Osteoporosis prevention, screening, and treatment: a review. J Womens Health 2014:23:563-72.

4 Ye C, Xu M, Wang S, et al. Decreased bone mineral density is an independent predictor for the development of atherosclerosis: a systematic review and metaanalysis. PLoS One 2016;11:e0154740.

5 Vassalle C, Mazzone A. Bone loss and vascular calcification: a bi-directional interplay? Vascul Pharmacol 2016;86:77-86.

6 Reiss AB, Miyawaki N, Moon J, et al. CKD, arterial calcification, atherosclerosis and bone health: inter-relationships and controversies. Atherosclerosis 2018;278:49-59.

7 Beer S, Saely CH, Hoefle G, et al. Low bone mineral density is not associated with angiographically determined coronary atherosclerosis in men. Osteoporos Int 2010;21:1695-701.

8 Brown HL, Warner JJ, Gianos E, et al. Promoting risk identification and reduction of cardiovascular disease in women through collaboration with obstetricians and Gynecologists: a presidential Advisory from the American heart association and the American College of obstetricians and Gynecologists. Circulation 2018;137:e843-52.

9 Kavousi M, Desai CS, Ayers C, et al. Prevalence and prognostic implications of coronary artery calcification in low-risk women: a meta-analysis. JAMA 2016:316:2126-34.
10 Navar-Boggan AM, Peterson ED, D'Agostino RB, et al. Using age- and sex-specific risk thresholds to guide statin therapy: one size may not fit all. J Am Coll Cardiol 2015;65:1633-9.

11 Yoo S, Lee KH, Lee HJ, et al. Seoul National University Bundang hospital's electronic system for total care. Healthc Inform Res 2012;18:145-52.

12 Farhat GN, Newman AB, Sutton-Tyrrell K, et al. The association of bone mineral density measures with incident cardiovascular disease in older adults. Osteoporos Int 2007;18:999-1008.

13 Fehérvári M, Sarkadi H, Krepuska M, et al. Bone mineral density is associated with site-specific atherosclerosis in patients with severe peripheral artery disease. Calcif Tissue Int 2013;93:55-61.

14 Bosworth C, Sachs MC, Duprez D, et al. Parathyroid hormone and arterial dysfunction in the multi-ethnic study of atherosclerosis. Clin Endocrinol 2013;79:429-36.

15 Shekarkhar S, Foroughi M, Moatamedi M, et al. The association of serum parathyroid hormone and severity of coronary artery diseases. Coron Artery Dis 2014;25:339-42.

16 Ajeganova S, Gustafsson T, Jogestrand T, et al. Bone mineral density and carotid atherosclerosis in systemic lupus erythematosus: a controlled cross-sectional study. Arthritis Res Ther 2015;17:84.

17 Jung KJ, Jang Y, Oh DJ, et al. The ACC/AHA 2013 pooled cohort equations compared to a Korean risk prediction model for atherosclerotic cardiovascular disease. Atherosclerosis 2015;242:367-75.

$18 \mathrm{Kim} \mathrm{KM}$, Moon JH, Choi SH, et al. Lower baseline value and greater decline in $\mathrm{BMD}$ as independent risk factors for mortality in community dwelling elderly. Bone 2019:121:204-11.

19 Schousboe JT, Shepherd JA, Bilezikian JP, et al. Executive summary of the 2013 International Society for clinical densitometry position development conference on bone densitometry. J Clin Densitom 2013;16:455-66.

20 Kanis JA. Assessment of osteoporosis at the primary health care level. who collaborating centre for metabolic bone diseases. University of Sheffield Medical School, 2008.

21 Alissa EM, Alnahdi WA, Alama N, et al. Bone mineral density and cardiovascular risk factors in postmenopausal women with coronary artery disease. Bonekey Rep 2015;4:758.

22 Yoon YE, Kim KM, Han JS, et al. Prediction of Subclinical Coronary Artery Disease With Breast Arterial Calcification and Low Bone Mass in Asymptomatic Women: Registry for the Women Health Cohort for the BBC Study. JACC Cardiovasc Imaging 2019:12:1202-11.

23 Lewis JR, Eggermont CJ, Schousboe JT, et al. Association between abdominal aortic calcification, bone mineral density, and fracture in older women. J Bone Miner Res 2019;34:2052-60.

24 Caffarelli C, Montagnani A, Nuti R, et al. Bisphosphonates, atherosclerosis and vascular calcification: update and systematic review of clinical studies. Clin Interv Aging 2017;12:1819-28.

25 Jiang Y, Fan Z, Wang Y, et al. Low bone mineral density is not associated with subclinical atherosclerosis: a population-based study in rural China. Cardiology 2018;141:78-87.

26 Störk S, von Schacky C, Angerer P. The effect of 17beta-estradiol on endothelial and inflammatory markers in postmenopausal women: a randomized, controlled trial. Atherosclerosis 2002;165:301-7.

27 Grundy SM, Stone NJ, Bailey AL, et al. 2018 AHA/ACC/AACVPR/AAPA/ABC/ACPM/ ADA/AGS/APhA/ASPC/NLA/PCNA guideline on the management of blood cholesterol: a report of the American College of Cardiology/American heart association Task force on clinical practice guidelines. Circulation 2019;139:e1082-143.

28 Yeboah J, McClelland RL, Polonsky TS, et al. Comparison of novel risk markers for improvement in cardiovascular risk assessment in intermediate-risk individuals. JAMA 2012;308:788-95.

29 Burgess S, Ference BA, Staley JR, et al. Association of LPA Variants With Risk of Coronary Disease and the Implications for Lipoprotein(a)-Lowering Therapies: A Mendelian Randomization Analysis. JAMA Cardiol 2018;3:619-27.

30 Choi YJ, Oh HJ, Kim DJ, et al. The prevalence of osteoporosis in Korean adults aged 50 years or older and the higher diagnosis rates in women who were beneficiaries of a national screening program: the Korea National health and nutrition examination survey 2008-2009. J Bone Miner Res 2012:27:1879-86. 